\title{
Construction of Infectious cDNA Clone of a Chrysanthemum stunt viroid Korean Isolate
}

\author{
Ju-Yeon Yoon ${ }^{1}$, In-Sook Cho ${ }^{2}$, Gug-Seoun Choi ${ }^{2}$ and Seung-Kook Choi ${ }^{2 *}$ \\ ${ }^{1}$ Department of Horticulture and Landscape, Seoul Women's University, Seoul 139-774, Korea \\ ${ }^{2}$ Department of Horticultural Environment, National Institute of Horticultural and Herbal Science, RDA 441-440, Korea
}

(Received on August 7, 2013; Revised on September 30, 2013; Accepted on September 30, 2013)

Chrysanthemum stunt viroid (CSVd), a noncoding infectious RNA molecule, causes seriously economic losses of chrysanthemum for 3 or 4 years after its first infection. Monomeric cDNA clones of CSVd isolate SK1 (CSVdSK1) were constructed in the plasmids pGEM-T easy vector and pUC19 vector. Linear positive-sense transcripts synthesized in vitro from the full-length monomeric cDNA clones of CSVd-SK1 could infect systemically tomato seedlings and chrysanthemum plants, suggesting that the linear CSVd RNA transcribed from the cDNA clones could be replicated as efficiently as circular CSVd in host species. However, direct inoculation of plasmid cDNA clones containing full-length monomeric cDNA of CSVd-SK1 failed to infect tomato and chrysanthemum and linear negative-sense transcripts from the plasmid DNAs were not infectious in the two plant species. The cDNA sequences of progeny viroid in systemically infected tomato and chrysanthemum showed a few substitutions at a specific nucleotide position, but there were no deletions and insertions in the sequences of the CSVd progeny from tomato and chrysanthemum plants.

Keywords : Chrysanthemum, Chrysanthemum stunt viroid, infectious cDNA, RT-PCR, tomato

Viroids are the smallest (246 to 401 nucleotides) selfreplicating RNAs capable of inducing a full-range of disease symptoms in susceptible host species, including potato, tomato, cucumber, hop, coconut, grapevine, fruit trees (avocado, peach, apple, pear, citrus, and plum), and a few flowering plant species (Flores et al., 2005). Genome of viroids consists of single-stranded, covalently

*Corresponding author.

Phone) +82-31-290-6236, FAX) +82-31-290-6259

E-mail) viroid73@gmail.com closed, circular and highly structured RNA and viroids exist as a circular structure with a high degree of selfcomplementarity, either to promote compact folding or to elicit their functions (Daros et al., 2006; Ding, 2009; Flores et al., 2005). These plant-pathogenic RNAs are unable to code for proteins, but it is fascinating that the short genomes of the viroids are able to contain all of the information related to intracellular trafficking, localization, replication, and pathogenicity, though their infection cycle is completely dependent on host factors (Flores et al., 2005; Tsagris et al., 2008). Since the discovery of Potato spindle tuber viroid (PSTVd; Diener, 1971) about 4 decades ago, approximately 30 species of viroids and their variants are known (Flores et al., 2005). Viroids are classified into two families: Pospiviroidae, whose replication take place in the nucleus, and Avsunviroidae, which replicates and accumulates in the chloroplast (Daros et al., 2006; Ding, 2009; Flores et al., 2005; Tsagris et al., 2008). These viroids are clearly distinguished by structural, functional, and biological properties. For instance, in Pospiviroidae viroids, the secondary structure of the RNA is either quasi-double-stranded or rod-like, whereas the RNA in Avsunviroidae viroids assumes highly branched secondary structures.

Chrysanthemum stunt viroid (CSVd) was first reported in chrysanthemum species (Dendranthema spp., formerly Chrysanthemum spp.) in 1947 at the time of rapid expansion of the cultivated chrysanthemum industry in the USA (Dimock, 1947), and by the 1950s, the disease had spread rapidly all over the world, affecting both the plants and flowers of different chrysanthemum cultivars (Lawson, 1987). CSVd causes seriously economic losses of chrysanthemum for 3 or 4 years after its first infection. Typical reactions in CSVd-infected chrysanthemum cultivars are stunting with a reduction of one-half to twothirds relative to the normal plant height and flower color bleaching (Bouwen and Annemarie, 1995; Diener and Lawson, 1973; Hooftman et al., 1996; Horst and Nelson, 
1997). The affected plants are often unproductive and the causal viroid is easily transmitted by foliar contact, cultivation practices and cutting knives.

Construction of full-length infectious cDNAs derived from various viroid strains has extensively used for infectivity, symptom evaluation, determination of pathogenicity domains, and movement of viroids (Cress et al., 1983; Chung et al., 2005; Kiefer et al., 1983; Matsushita and Penmetcha, 2009; Meshi et al., 1984; Navarro and Flores, 1997; Verhoeven et al., 2009; Visvader and Symons, 1986; Zhu et al., 2002). Here, we describe the construction of plasmids containing full-length monomeric cDNA of a CSVd Korean isolate (named CSVd-SK1) as well as show that transcripts from the cDNA plasmids are infectious to viroid-free chrysanthemum as well as tomato. We also examined the sequences for whether there are any alterations in progeny viroid from the infected chrysanthemum and tomato plants.

\section{Materials and Methods}

Viroid source and cDNA synthesis. A chrysanthemum showing distinct stunting symptoms was collected from a greenhouse in 2011, in Chung-Nam province, Korea. To detect CSVd infection from the diseased chrysanthemum plant, total RNA was extracted from $5 \mathrm{~g}$ of frozen $\left(-80^{\circ} \mathrm{C}\right)$ leaves of the infected chrysanthemum using Plant RNeasy Mini kit (Qiagen, USA), according to the manufacturer's instructions. Based on the sequences of CSVd isolate K1 (Genbank accession no. AF394452), One-step RT-PCR analysis was carried out to synthesize full-length cDNA of CSVd using CSVd-specific primers [CSVd-For: 5'-aaagaaatgaggegaagaag-3' and CSVdRev: 5'-ttcttcaaagcagcagggt-3'], as described previously (Chung et al., 2001). The thermo-cycling conditions were as follows: $60 \mathrm{~min}$ at $50^{\circ} \mathrm{C}$ for reverse transcription (RT), $5 \mathrm{~min}$ at $95^{\circ} \mathrm{C}(1 \mathrm{cycle}), 94^{\circ} \mathrm{C}, 30 \mathrm{~s}, 53^{\circ} \mathrm{C}, 30 \mathrm{~s}$ and $72^{\circ} \mathrm{C}$, $30 \mathrm{~s}$ (40 cycles), and a final extension at $72^{\circ} \mathrm{C}$ for $7 \mathrm{~min}$. RT-PCR product was analyzed in $1.2 \%$ agarose gel and visualized after soaking in ethidium bromide solution.

Cloning and sequence analysis of the isolated CSVd. RT-PCR product synthesized from the diseased chrysanthemum was directly cloned into the pGEM-T easy vector (Promega, USA) resulting in pGEM-CSVd-SK1. This cDNA clone was used to determine sequences of the entire insert cDNA, using BigDye terminator cycle sequencing kit (Applied Biosystems, USA) in both directions, according to manufacturer's instructions. The determined nucleotide sequence of CSVd-SK1 was analyzed using DNASTAR software and BLAST search.
Construction of infectious cDNA clone and in vitro transcription. To minimize non-viroid sequence in full-length monomeric cDNA clone of CSVd-SK1, the insert in pGEMCSVd-SK1 was amplified with Xba-SP6-CSVd primer [5'-tc tagaatttaggtgacactatagaaagaaatgaggcgaagaag -3'; XbaI site (in bold), SP6 RNA polymerase promoter (underline) and 20 nucleotides identical to CSVd] and EcoR-CSVd-T7 primer [5'-taatacgactcactataggggaattcttcttcaaagcagcagg gt-3'; T7 RNA polymerase promoter (underline), EcoRI (in bold), and 20 nucleotides complementary to CSVd] using PfuUltra High-Fidelity DNA Polymerase, according to manufacturer's instructions (Agilent Technologies, USA). The synthesized PCR product was digested with $X b a \mathrm{I}$ and EcoRI enzymes, and subsequently the $X b a \mathrm{I}-E c o$ RI fragment was cloned in pUC19 digested with the same restriction enzymes, resulting in pCSVd-SK1 (Fig. 1). The 354bp double-stranded cDNA of CSVd-SK1 was cloned from immediately between and adjacent to the SP6 and T7 promoter sequences. Transcripts of both polarities were produced using SP6 and T7 RNA polymerases after linearization of pGEM-CSVd-SK1 and pCSVd-SK1 with restriction enzymes, as described previously (Choi et al., 2003).

To prepare the positive sense of CSVd-SK1 [CSVdSK1(+)], pGEM-CSVd-SK1 containing full-length cDNA of CSVd-SK1 was used as templates for in vitro transcription. The plasmid was digested with SacII to produce a linear template DNA and blunted with Klenow fragment to remove non-viroid sequences, as described previously (Choi et al., 2005). In case of pCSVd-SK1, the cDNA clone was digested with EcoRI and further purified with ethanol precipitation. Infectious CSVd RNA was generated by in vitro transcription with SP6 RNA polymerase (Promega, USA) in the presence of and in the absence of the cap analogue $\mathrm{m}^{7} \mathrm{GpppG}$ (New England Biolabs, USA) following a procedure described previously (Canto and Palukaitis, 1998). To prepare the negative sense of CSVdSK1 [CSVd-SK1(-)], pGEM-CSVd-SK1 was digested with SpeI to produce a linear template DNA. Sub- sequent procedures for preparation of capped or uncapped CSVdSK1(-) transcripts from pCSVd-SK1 digested with $X b a \mathrm{I}$ were identical via T7 RNA transcription as described above.

CSVd-SK1(+) and CSVd-SK1(-) RNAs from the clones were mechanically inoculated to chrysanthemum (cultivar Vivid Scarlette, Korea) and tomato seedlings (cultivar PpoTto, Korea) with Carborundum ${ }^{\circledR}$ (Thermo Scientific, USA), along with the PCR products, cDNA fragments from the plasmids and the plasmids themselves (pGEM-CSVd-SK1 and pCSVd-SK1). CSVd RNAs isolated from the infected chrysanthemum were used as a positive control and $0.5 \mathrm{X}$ 


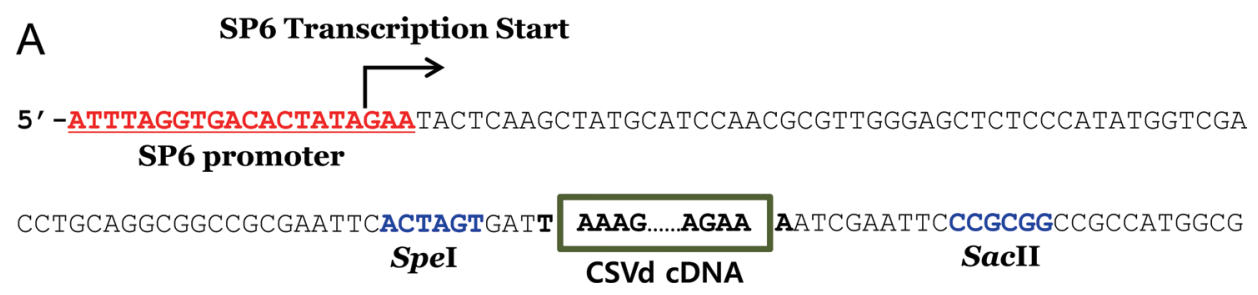

GCCGGGAGCATGCGACGTCGGGCCCAATTCGCCCTATAGTGAGTCGTATTA

T7 Transcription Start

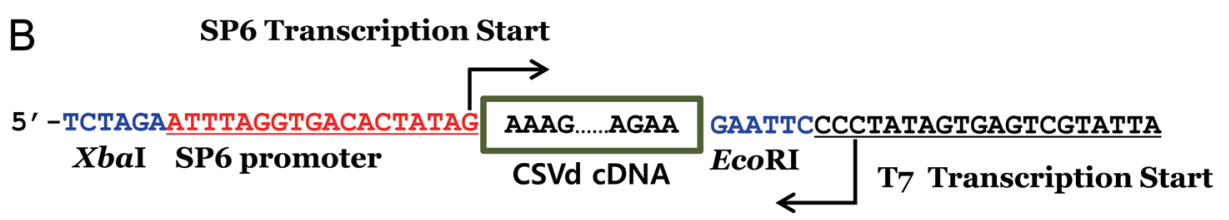

Fig. 1. Construction of full-length monomeric cDNA clones of CSVd-SK1. (A) Schematic sequences of both vector and cDNA insert of CSVd-SK1 in pGEM-CSVd-SK1. SP6 promoter site (red and underline) and SacII site (blue) were used for the synthesis of positivesense transcript of CSVd-SK1. T7 promoter site (black and underline) and SpeI site (blue) were used for the production of negative-sense transcript of CSVd-SK1. The CSVd-SK1 cDNA is presented as a box. (B) Schematic nucleotide sequence of the cDNA insert of CSVdSK1 in pCSVd-SK1. SP6 promoter site (red and underline) and EcoRI site (blue) were used for the synthesis of positive-sense transcript of CSVd-SK1. T7 promoter site (black and underline) and XbaI site (blue) were used for the production of negative-sense transcript of CSVd-SK1. SP6 and T7 Transcription start sites are indicated by rectangle arrows.

PBS buffer was used as a negative control. The inoculated plants were propagated in a growth chamber $\left(25^{\circ} \mathrm{C}\right)$ under a long photoperiod (18 hours).

Analysis of CSVd infection and sequencing of CSVd progeny. Leaves $(0.1 \mathrm{~g})$ of CSVd-infected chrysanthemum and tomato plants were ground in buffer $\mathrm{B}(0.1 \mathrm{M}$ Tris- $\mathrm{HCl}$, $\mathrm{pH}$ 9.0, $5 \mathrm{M}$ urea, 0.1 M NaCl, 0.1 M 2-mercaptoethanol, and $10 \mathrm{mM}$ EDTA). The homogenate was clarified by centrifugation, and the supernatant was extracted with 0.5 vol of phenol/chloroform (1:1). Total RNA was recovered by ethanol precipitation and resuspended in sterile water. RT-PCR analysis for CSVd progeny was carried out as described above and the resulting RT-PCR fragment (fulllength) was cloned into pCR4TOPO vector (Invitrogen, USA). The entire cDNA insert was sequenced using a BigDye termination cycle sequencing kit (Applied Biosystems, USA) with M13 forward/reverse primers. The determined nucleotide sequences comparisons were calculated using the Jukes and Cantor index in the MEGA 5.0 Software (Choi et al., 2011).

\section{Results and Discussion}

To identify the sequence of CSVd-SK1 from an infected chrysanthemum plant, total purified RNA was subjected to
RT-PCR analysis using primers specific to CSVd strains. RT-PCR product was synthesized from the diseased chrysanthemum and the size of RT-PCR product expected was $354 \mathrm{bp}$ long. To determine the genome sequence of the detected CSVd-SK1, the synthesized RT-PCR product was directly introduced into the pGEM-T easy vector (Promega, USA), resulting in pGEM-CSVd-SK1. Sequencing analysis showed the genome sequence of CSVd-SK1 was truly $354 \mathrm{bp}$ in length. CSVd-SK1 shared 88 to $100 \%$ sequence identity with other CSVd isolates and $78-80 \%$ sequence identity with tomato apical stunt viroid isolates. CSV-SK1 shares $69-73 \%$ nucleotide identity with PSTVd and $67-69 \%$ sequence identity with Citrus exocortis viroid. These results suggest that CSVd-SK1 is a typical isolate from chrysanthemum species. The complete nucleotide sequences of CSVd-SK1 determined have been deposited in NCBI Genbank under accession number AB679193.

To eliminate non-viroid sequence from clone of CSVdSK1, pCSVd-SK1 harboring the full-length monomeric cDNA of CSVd-SK1 was constructed (Fig. 1). The clone allows synthesizing transcripts using SP6 or T7 RNA polymerase, resulting in the full-length transcripts of defined polarity (Fig. 1). We initiated a series of experiments to determine whether the double-stranded plasmid DNAs or the RNAs of different polarities synthesized from in vitro transcription were infectious when inoculated onto 
Table 1. Infectivity of Chrysanthemum stunt viroid RNAs in chrysanthemum and tomato plants

\begin{tabular}{|c|c|c|}
\hline \multirow{2}{*}{ Inoculum } & \multicolumn{2}{|l|}{ Plants } \\
\hline & Chrysanthemum & tomato \\
\hline pGEM-CSVd-SK1 & $0 / 10^{\mathrm{a}}$ & $0 / 10$ \\
\hline pCSVd-SK1 & $0 / 10$ & $0 / 10$ \\
\hline cDNA fragment of CSVd-SK1 & $0 / 10$ & $0 / 10$ \\
\hline CSVd-SK1(+)G & $6 / 10$ & $6 / 10$ \\
\hline CSVd-SK1(-)G & $0 / 10$ & $0 / 10$ \\
\hline CSVd-SK1(+)P & $9 / 10$ & $9 / 10$ \\
\hline CSVd-SK1(-)P & $0 / 10$ & $0 / 10$ \\
\hline Purified CSVd-SK1 RNAs ${ }^{\mathrm{b}}$ & $10 / 10$ & $9 / 10$ \\
\hline Mock & $0 / 10$ & $0 / 10$ \\
\hline \multicolumn{3}{|c|}{$\begin{array}{l}\text { "Number of plants showing systemic infection of CSVd/number of } \\
\text { plants inoculated }\end{array}$} \\
\hline
\end{tabular}

tomato seedlings and CSVd-free chrysanthemum plants. To differentiate the origin and polarity of CSVd-SK1 transcripts, we denote CSVd-SK1 transcripts as follows; for instance, the positive-sense transcript of CSVd-SK1 derived from pGEM-CSVd-SK1 clone is named CSVd$\mathrm{SK} 1(+) \mathrm{G}$ and the equivalent transcript from pCSVdSK1 clone is named CSVd-SK1(+)P. Infectivity was assayed by RT-PCR analysis of leaf sap prepared from the bioassay plants and by the appearance of disease symptoms simultaneously in 8 weeks.

Table 1 summarizes the results obtained with 2 different CSVd-SK1 cDNA clones and their corresponding RNA preparations. Only CSVd-SK1 transcripts [named CSVd$\mathrm{SK}(+) \mathrm{G}$ and CSVd-SK(+)P] containing positive-sense polarity replicated/moved in chrysanthemum and tomato plants with efficiency similar to that of the RNAs isolated from the original inoculum-source chrysanthemum infected naturally with CSVd-SK1. The presence of CSVd RNAs in upper (non-inoculated) leaves of the two plant species was detected by RT-PCR analysis at 3 week postinoculation (Fig. 2). Infectivity of capped CSVd-SK1(+) $\mathrm{G}$ and capped CSVd-SK1(+)P in chrysanthemum and tomato was very similar to infectivity of uncapped CSVd$\mathrm{SK}(+) \mathrm{G}$ and uncapped CSVd-SK(+)P. Neither capped CSVd-SK1(-)G nor capped CSVd-SK1(-)P could not infect chrysanthemum and tomato plants. Similar results were observed from infectivity tests with uncapped CSVdSK1(-)G and uncapped CSVd-SK1(-)P (data not shown). It is well known that structures found in the both termini of viral RNAs (i.e. cap structure pseudoknots) play important roles in the process of virus replication/movement through

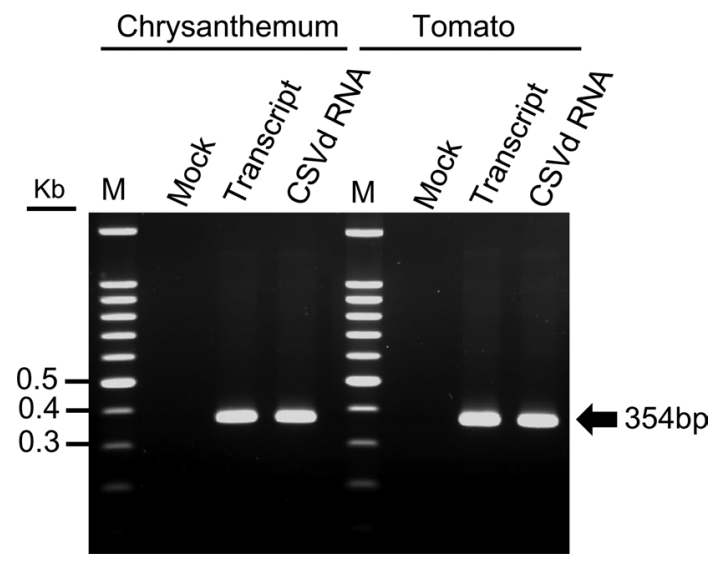

Fig. 2. RT-PCR analysis of transcripts from the cDNA clones of CSVd-SK1 in chrysanthemum and tomato plants. Total RNAs were extracted from upper (non-inoculated) leaves of chrysanthemum and tomato plants. RT-PCR was analyzed with CSVd-specific primers [CSVd-For: 5'-aaagaaatgaggcgaagaag-3' and CSVd-Rev: 5'-ttctttcaaagcagcagggt-3'], as described previously (Chung et al., 2005). The CSVd-SK1 RNAs purified from the infected chrysanthemum plant were used as a positive control and total RNA from mock inoculation (only with $0.5 \mathrm{X}$ PBS buffer) was used as a negative control. RT-PCR products were visualized in $1.2 \%$ agarose gel.

enhancement of viral RNA stability (Hull, 2009). Because CSVd replicates in vivo in the nucleus via an asymmetric rolling circle mechanism, it requires a specific cleavage procedure that converts multimeric viroid copies into monomeric forms (Flores et al., 2000; 2005). As for CSVd infection in chrysanthemum and tomato, our results suggest that the presence of cap structure at 5 ' end of linear CSVd RNAs is not prerequisite for the infections of host plants.

As "uncapped" positive-sense CSVd transcripts are sufficiently used as inocula onto chrysanthemum and tomato plants, hereafter, we did all experiments with "uncapped" CSVd transcripts containing defined polarity. It is noteworthy that CSVd-SK(+)P containing the minimized non-viroid sequence showed higher infectivity than CSVdSK $(+) \mathrm{G}$ that contains some non-viroid sequence (Table 1) and that the infectivity of CSVd-SK(+)P in chrysanthemum and tomato plants was similar to that of the wild-type CSVd-SK1 RNAs purified from the inoculum-source chrysanthemum (Table 1). The data presented in Table 1 are similar to results from infectivity assay using in vitrotranscribed RNAs of CSVd Japan isolate (Matsushita and Penmetcha, 2009). Table 1 shows that the infectivity of the linear as well as the circular form of CSVd is intriguing as similar results have been obtained for CSVd Australian isolate (Palukaitis and Symons, 1980) and for PSTVd (Owens et al., 1977). It is interesting that positive-sense 
monomeric RNAs of CSVd-SK1 are highly infectious, because transcribed RNAs that consist of monomeric sequence of PSTVd showed low infectivity (Cress et al., 1983).

DNA infectivity was not observed for the monomeric cDNA clones pGEM-CSVd-SK1 and pCSVd-SK1 in chrysanthemum and tomato plants (Table 1). To exclude the possibility that failure of direct DNA infectivity with the cDNA clones were caused by supercoiled plasmid DNAs from E. coli, the clones were digested with restriction enzymes which cleave the plasmid vector sequences outside the cDNA insert of CSVd-SK1. The monomeric cDNA fragments of CSVd-SK1 from the digested clones failed to infect the two plant species. Similarly, the amplified PCR products from pGEM-CSVd-SK1 and pCSVd-SK1 could not infect chrysanthemum and tomato plants. Therefore, the data presented in Table 1 showed that the DNA infectivity of CSVd-SK1 was not significantly affected by vector sequence adjacent to CSVd cDNA sequence and its topological nature. In the case of PSTVd, DNA infectivity was consistently observed for two dimeric cDNA clones of PSTVd containing head-totail positions, while no infection was obtained from any of the monomeric cDNA clones of PSTVd in tomato plants (Cress et al., 1983). Interestingly, It was found that not only double-stranded but also all M13-cloned dimeric and multimeric single-stranded PSTVd cDNAs were infectious irrespective of whether they represent the PSTVd $(+)$ or (-) cDNA strand (Tabler and Sänger, 1984). The vectorinserted monomeric PSTVd cDNA units were also found to be infectious but of low specific infectivity which was increased when these monomers had been excised. Even two subgenomic DNA fragments, representing together the 359 nucleotides of the PSTVd RNA genome, initiated the synthesis of viroid RNA progeny when co-inoculated although each fragment by itself is non-infectious (Tabler and Sänger, 1984). In contrast to the case that monomeric double-stranded cDNA of PSTVd (Cress et al., 1983) was non-infectious, DNA infectivity was successful for monomeric cDNA clones of Hop stunt viroid (HSVd) in cucumber (Meshi et al., 1984), though it is not known in detail how these cDNAs initiate the infection process of HSVd. It remains to be determined whether direct DNA inoculations with dimeric or trimeric cDNA clones of CSVd will infect chrysanthemum and tomato plants.

At 8 weeks post-inoculation, chrysanthemum plants inoculated with CSVd-SK1(+)G or CSVd-SK1(+)P showed stunting, a typical symptom of CSVd, compared with the uninfected chrysanthemum (Fig. 3A). In contrast, the transcripts CSVd-SK1(+)G and CSVd-SK1(+)
A

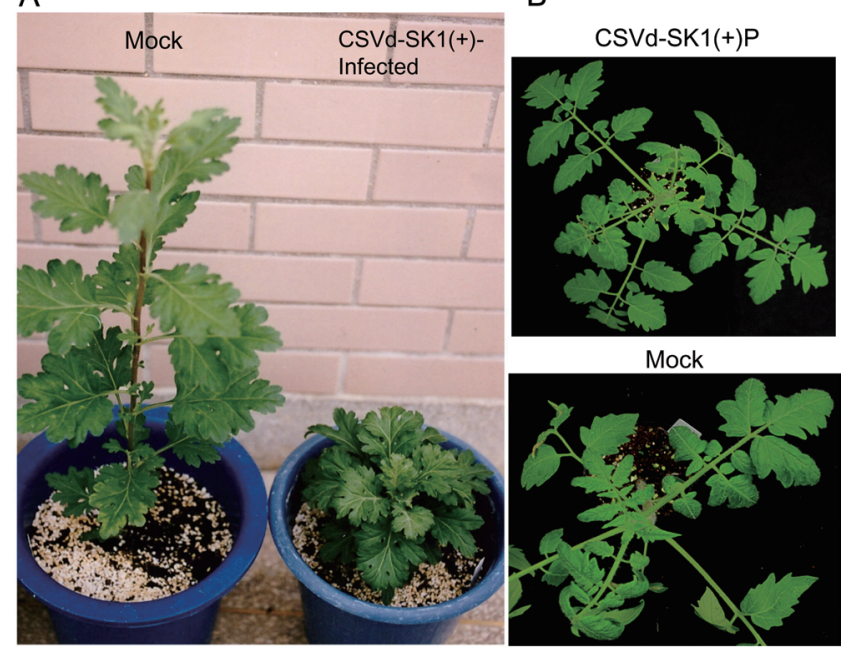

Fig. 3. Symptoms of chrysanthemum and tomato plants induced by CSVd-SK1(+) transcripts. (A) Stunting symptom of chrysanthemum infected by CSVd-SK1(+)P and mock means mechanical inoculation with $0.5 \mathrm{X}$ PBS buffer alone. (B) No distinct symptoms of tomato plants infected by CSVd-SK1(+) P. The CSVd Infection from the symptomless tomato plants was confirmed by RT-PCR analysis.

$\mathrm{P}$ did not show any visible symptoms in tomato plants, similar to CSVd-SK1 RNAs purified from the infected chrysanthemum plant (Fig. 3B). RT-PCR analysis from the leaf samples of inoculated tomato verified that transcripts of CSVd-SK1 $(+)$ infected tomato plants systemically (Fig. $2)$. These results suggest that the linear positive-sense RNAs of CSVd-SK1 [CSVd-SK1(+)G and CSVd-SK1(+) $\mathrm{P}]$ are efficiently replicated and transported throughout plants via vascular system and full-length monomeric $(+)$ RNA of CSVd is sufficient for successful infections in chrysanthemum and tomato plants, similar to results that have been obtained previously for CSVd Japan isolate (Matsushita and Penmetcha, 2009).

To address whether the replicated transcript retained its primary sequence or underwent nucleotide substitutions or deletions, we amplified and sequenced $30 \mathrm{CSVd}$ clones from each infected chrysanthemum and tomato plants and compared the primary sequences of CSVd-SK1 using computational analysis tools. No substitutions or deletions in the primary sequence of CSVd-SK1 were observed in the progeny RNAs of CSVd-SK1(+) from the chrysanthemum isolate (Table 2). However, a few substitutions, primarily at nucleotide position $49(\mathrm{G} \rightarrow \mathrm{A}$; purine transition) were observed when the inoculated RNA was analyzed from the infected tomato plants. The nucleotide substitution of G49A was one of the nucleotide positions that showed the highest level of sequence 
Table 2. Infectivity of Chrysanthemum stunt viroid RNAs in chrysanthemum and tomato plants

\begin{tabular}{|c|c|c|c|c|c|c|}
\hline \multirow{3}{*}{ Inoculum } & \multicolumn{6}{|c|}{ Nucleotide substitution } \\
\hline & \multicolumn{3}{|c|}{ Chrysanthemum } & \multicolumn{3}{|c|}{ Tomato } \\
\hline & Sample1 & Sample2 & Sample3 & Sample1 & Sample2 & Sample3 \\
\hline CSVd-SK1(+)G & $\begin{array}{l}0 / 30 \\
\left(-{ }^{b}\right)\end{array}$ & $\begin{array}{c}0 / 30 \\
(-)\end{array}$ & $\begin{array}{c}0 / 30 \\
(-)\end{array}$ & $\begin{array}{c}1 / 30 \\
\left(\mathrm{G} 49 \mathrm{~A}^{\mathrm{C}}\right)\end{array}$ & $\begin{array}{c}2 / 30 \\
(\mathrm{G} 49 \mathrm{~A})\end{array}$ & $\begin{array}{c}1 / 30 \\
(\mathrm{G} 49 \mathrm{~A})\end{array}$ \\
\hline CSVd-SK1(+)P & $\begin{array}{l}0 / 30 \\
(-)\end{array}$ & $\begin{array}{c}0 / 30 \\
(-)\end{array}$ & $\begin{array}{c}0 / 30 \\
(-)\end{array}$ & $\begin{array}{c}1 / 30 \\
(\mathrm{G} 49 \mathrm{~A})\end{array}$ & $\begin{array}{c}1 / 30 \\
(\mathrm{G} 49 \mathrm{~A})\end{array}$ & $\begin{array}{c}1 / 30 \\
(\mathrm{G} 49 \mathrm{~A})\end{array}$ \\
\hline $\begin{array}{l}\text { Purified CSVd-SK1 } \\
\text { RNAs }^{\mathrm{a}}\end{array}$ & $\begin{array}{c}0 / 30 \\
(-)\end{array}$ & $\begin{array}{c}0 / 30 \\
(-)\end{array}$ & $\begin{array}{c}0 / 30 \\
(-)\end{array}$ & $\begin{array}{c}1 / 30 \\
(\mathrm{G} 49 \mathrm{~A})\end{array}$ & $\begin{array}{c}1 / 30 \\
(\mathrm{G} 49 \mathrm{~A})\end{array}$ & $\begin{array}{c}2 / 30 \\
(\mathrm{G} 49 \mathrm{~A}) \\
(\mathrm{U} 47 \mathrm{~A})\end{array}$ \\
\hline
\end{tabular}

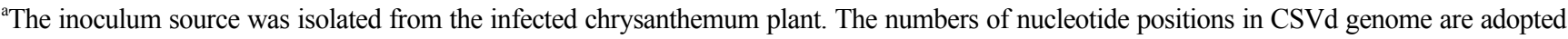
from the previous published

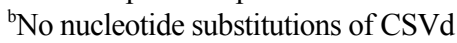

"Number of cDNAs showing nucleotide substitutions of CSVd/total number of clones sequenced

variation, which were located at positions $47,49,50,64$, 65, 254 and 298 in the CSVd populations though sequence variation was observed at 103 sites scattered though the CSVd genone (Yoon and Palukaitis, 2013). Our sequence analyses of the CSVd-SK1 isolated from two host species suggest that the CSVd isolates are likely to change some nucleotides in their genomes when the CSVd isolates replicate, depending on the host species (Gross et al., 1982; Matsushita and Penmetcha, 2009). We could not exclude completely the possibility that consecutive passages of CSVd from the cDNA clones of CSVd-SK1 give rise to substitutions, deletions, and insertions in the genome of CSVd in natural host species, such as chrysanthemum, tomato, petunia, pepper and potato (Murcia et al., 2011). Whether a series of passages for CSVd in specific host species affects infectivity by sequence variations remains to be determined.

\section{Acknowledgements}

This work was supported by a grant from the Next Generation BioGreen21 program (Project no. PJ007984032013), Rural Development Administration, Republic of Korea.

\section{References}

Bouwen, I. and Annemarie, Z. 1995. Chrysanthemum. In : Virus and virus-like diseases of bulb and flower crops, eds. by G. Loebenstein, R. H. Lawson and A. A. Brunt, pp. 396-408. John wiley \& sons, New York, USA.

Canto, T. and Palukaitis, P. 1998. Transgenically expressed cucumber mosaic virus RNA 1 simultaneously complements replication of cucumber mosaic viruses RNA 2 and 3 and confers resistance to systemic infection. Virology 250:325336.

Choi, S. K., Choi, J. K. and Ryu, K. H. 2003. Involvement of RNA2 for systemic infection of Cucumber mosaic virus isolated from lily on zucchini squash. Virus Res. 97:1-6.

Choi, S. K., Palukatis, P., Min, B. E., Lee, M. Y., Choi, J. K. and Ryu, K. H. 2005. Cucumber mosaic virus 2 a polymerase and 3 a movement proteins independently affect both virus movement and the timing of symptom development in zucchini squash. J. Gen. Virol. 86:1213-1222.

Choi, S. K., Yoon, J. Y., Canto, T. and Palukaitis, P. 2011. Replication of cucumber mosaic virus RNA 1 in cis requires functional helicase-like motifs of the 1a protein. Virus Res. 158:271-276.

Chung, B. N., Lim, J. H., Choi, S. Y., Kim, J. S., and Lee, E. J. 2005. Occurrence of Chrysanthemum stunt viroid in chrysanthemum in Korea. Plant Pathol. J. 21:377-382.

Cress, D. E., Kiefer, M. C. and Owens, R. A. 1983. Construction of infectious Potato spindle tuber viroid cDNA clones. Nucleic Acids Res. 11:6821-6835.

Daròs, J. A., Elena, S. F. and Flores, R. 2006. Viroids: Ariadne's thread into the RNA labyrinth. EMBO Rep. 7:593-598.

Diener, T. O. 1971. Potato spindle tuber "virus". IV. A replicating, low molecular weight RNA. Virology 45:411-428.

Diener, T. O. and Lawson, R. H. 1973. Chrysanthemum stunt: a viroid disease. Virology 51:94-100.

Dimock, A. W. 1947. Chrysanthemum stunt. N.Y. State Flower Grow. Bull. 26:2.

Ding, B. 2009. The biology of viroid-host interactions. Annu. Rev. Phytopathol. 47:105-131.

Flores, R., Daròs, J. A. and Hernández, C. 2000. Avsunviroidae family: Viroids containing hammerhead ribozymes. $A d v$. Virus Res. 55:271-323.

Flores, R., Hernández, C., Martínez de Alba, A. E., Daròs, J. A. and Di Serio F. 2005. Viroids and viroid-host interactions. 
Annu. Rev. Phytopathol. 43:117-139.

Gross, H. J., Krupp, G., Domdey, H., Raba, M., Alberty, H., Lossow, C. H., Ramm, K. and Sänger, H. L. 1982. Nucleotide sequence and secondary structure of citrus exocortis and Chrysanthemum stunt viroid. Eur. J. Biochem. 121:249-257.

Hooftman, R., Arts, M.-J., Shamloul, A.M., Zaayen, A. van and Hadidi, A. 1996. Detection of Chrysanthemum stunt viroid by reverse transcription-polymerase chain reaction and by tissue blot hybridization. Acta Hort. 432:120-129.

Horst, R. K. and Nelson, P. E. 1997. Compendium of Chrysanthemum Diseases. APS Press, St, USA.

Hull, R. 2009. Comparative Plant Virology, $2^{\text {nd }}$ Ed. London, Elsevier Academic Press.

Kiefer, M. C., Owens, R. A. and Diener, T. O. 1983. Structural similarities between viroids and transposable genetic elements. Proc. Natl. Acad. Sci. USA 80:6234-6238.

Lawson, R. H. 1987. Chrysanthemum stunt. In The Viroids, ed. by T.O. Diener, pp 247-259. springer, Gemany.

Matsushita, Y. and Penmetcha, K. K. R. 2009. In vitro-transcribed chrysanthemum stunt viroid RNA is infectious to chrysanthemum and other plants. Phytopathology 99:58-66.

Meshi, T., Ishikawa, M., Ohno, T., Okada, Y., Sano, T., Ueda, I. and Shikata, E. 1984. Double-stranded cDNAs of Hop stunt viroid are infectious. J. Biochem. 95:1521-1524.

Murcia, N., Bernad, L., Duran-Vila, N. and Serra, P. 2011. Two nucleotide positions in the Citrus exocortis viroid RNA associated with symptom expression in Etrog citron but not in experimental herbaceous hosts. Mol. Plant Pathol. 12:203208.

Navarro, B. and Flores, R. 1997. Chrysanthemum chlorotic mottle viroid: unusual structural properties of a subgroup of self-cleaving viroids with hammerhead ribozymes. Proc. Natl. Acad. Sci. USA 94:11262-11267.

Owens, R. A., Erbe, R., Hadidi, A., Steere, R. I. and Diener, T. O. 1977. Infection of tomato protoplasts by ribonucleic acid of Tobacco mosaic virus and by viroids. Phytopathol. Zeitschrift 90:289-305.

Palukaitis, P. and Symons, R. H. 1980. Purification and characterization of the circular and linear forms of Chrysanthemum stunt viroid. J. Gen. Virol. 46:477-489.

Tabler, M. and Sänger, H. L. 1984. Cloned single- and doublestranded DNA copies of Potato spindle tuber viroid (PSTVd) RNA and co-inoculated subgenomic DNA fragments are infectious. EMBO J. 3:3055-3062.

Tsagris, E. M., Martinez de Alba, A. E., Gozmanova, M. and Kalantidis, K. 2008. Viroids. Cell Microbiol. 10:2168-2179.

Verhoeven, J. Th. J., Jansen, C. C. C., Roenhorst, J. W., Flores, R. and De la Penã, M. 2009. Pepper chat fruit viroid: biological and molecular properties of a proposed new species of the genus Pospiviroid. Virus Res. 144:209-214.

Visvader, J. E. and Symons, R. H. 1986. Replication of in vitro constructed viroid mutants: location of the pathogenicity modulating domain of citrus exocortis viroid. EMBO J. 5:2051-2055.

Yoon, J. Y. and Palukaitis, P. 2013. Sequence comparisons of global Chrysanthemum stunt viroid variants: multiple polymorphic positions scattered through the viroid genome. Virus Genes 46:97-104.

Zhu, Y. Qi, Y., Xun, Y., Owens, R. and Ding, B. 2002. Movement of Potato spindle tuber viroid reveals regulatory points of phloem-mediated RNA traffic. Plant Physiol. 130:138-146. 